\title{
Human Brucellosis: Risks and Prevalence among Iranian Blood Donors Residing in Endemic Areas
}

\author{
Maryam Zadsar ${ }^{\mathrm{a}}$ Mohammad Reza Shirzadi ${ }^{\mathrm{b}}$ Mohammad Zeynali ${ }^{\mathrm{b}}$ \\ Mahboubeh Rasoulic Gharib Karimi ${ }^{\mathrm{a}}$ \\ a Blood Transfusion Research Centre, High Institute for Research and Education in Transfusion Medicine, Tehran, \\ Iran; ${ }^{b}$ Centre for Disease Control, Ministry of Health, Tehran, Iran; ${ }^{\mathrm{c} D e p a r t m e n t}$ of Biostatics, School of Public Health, \\ Iran University of Medical Sciences, Tehran, Iran
}

\section{Keywords}

Blood donation $\cdot$ Brucella $\cdot$ Donors $\cdot$ Infection

\begin{abstract}
Background: Brucellosis is a common zoonotic infection worldwide. Transmission can be occasionally observed via transfusion or transplantation. This study was designed to survey the seroprevalence of anti-Brucella antibody in blood donors in different endemic provinces. Materials and Methods: A total of 14,706 blood donors from the 5 most prevalent provinces were studied by standard tube agglutination (STA) and any positive subjects were further confirmed by 2-mercaptoethanol agglutination test (2-ME). Significant titres were 80 for STA and 40 for 2-ME. Result: A total of 63 $(0.43 \%)$ serum samples were STA-positive. Of these, the $2-\mathrm{ME}$ test was reactive in 42 samples. The $2-M E$ test was reactive in 31 samples with a low titre. However, concomitant STA $\geq 80$ and the titre of $\geq 40$ for the $2-M E$ test were found in 11 subjects $(0.075 \%)$, mostly resident in urban areas. Exposure to manure products was identified as a significant risk factor ( $p=0.0128)$. Conclusion: The observed data show a somewhat noticeable prevalence among Iranian blood donors, bringing attention to the importance of pre-donation screening via a questionnaire which supplies answers about occupational history, and any history of exposure or past infections. Further studies to evaluate the frequency and related risk factors in certain populations, in conjunction with implementing stricter regulations in blood donor selection in endemic areas, may be necessary. @ 2019 S. Karger AG, Basel
\end{abstract}

-Mail ghkarimi37@yahoo.com cellosis by David Bruce in 1887, and the establishment of the zoonotic nature of the disease by Zammit in 1905 [1], features of the disease have changed drastically due to many sanitary and socioeconomic factors. However, brucellosis is still a common zoonotic infection, with nearly 500,000 new cases of human brucellosis occurring annually worldwide [2]. It is recognised as a serious public health problem in the eastern Mediterranean region in which Iran is located. A recently published systematic reiew reported that the pooled annual incidence of brucelsis in Iran might be 1/10,000, and that the highest inciins of the country [3]. The most common transmission domestic animal products. Direct human-to-huor transplantation are also reported. Brucella melitensis, cause cause protean disease with a wide range of clinical presention in from asymptomatic donors [7]. A case of brucellosis after liver transplantation was reported 2 months post-operatively in Turkey [8]. Akçakuş et al. [9] reported the transmission of Brucella after exchange transfusion in 2 new- 
Table 1. Geographical origin of blood donors and Brucella seroprevalence

\begin{tabular}{|c|c|c|c|c|c|}
\hline Province & $\begin{array}{l}\text { Prevalence in general } \\
\text { population/1,000 }\end{array}$ & $\begin{array}{l}\text { Number of blood } \\
\text { donations }\end{array}$ & $\begin{array}{l}\text { Study blood donors, } \\
n\end{array}$ & $\begin{array}{l}\text { Seropositive blood donors, } \\
n\end{array}$ & $\begin{array}{l}\text { Seroprevalence in } \\
\text { blood donors } / 1,000\end{array}$ \\
\hline Hamedan & 0.66 & 40,225 & 2,651 & 49 & 18.4 \\
\hline Kurdistan & 0.79 & 25,743 & 2,352 & 0 & 0 \\
\hline Ardabil & 0.38 & 34,921 & 2,835 & 3 & 1.05 \\
\hline Eastern Azerbaijan & 0.42 & 95,830 & 4,876 & 3 & 0.6 \\
\hline Zanjan & 0.55 & 23,207 & 1,992 & 8 & 4 \\
\hline Total & 0.23 & 219,929 & 14,706 & 63 & 4.2 \\
\hline
\end{tabular}

borns with positive blood cultures. Another case suggesting post-transfusion brucellosis in a premature infant manifested as low-grade fever, decreased activity, poor weight gain, and positive blood culture [10].

Whole-blood products in Iran meet the standards of healthy blood donor recruitment and selection, in addition to a negative result when screening for HIV, hepatitis B virus (HBV), HCV, syphilis, and human T-lymphotropic virus (HTLV; in endemic regions). The current leukoreduction process is only performed on blood products which are ordered for special cases such as thalassemia patients.

It may become necessary to evaluate the safety-guard of Brucella transmission via blood products, particularly in endemic areas because only a low infectious dose of Brucella spp. is needed to induce human disease. Although currently no minimum infectious dose via blood products exists, an infectious level of the bacteria of 10 100 microorganisms has been estimated from animal experiments $[11,12]$. The long incubation period creates clinical and laboratory diagnostic challenges, and the effect of the leukoreduction procedure on preventing transfusion transmission remains unknown [13]. Given the high prevalence of infection and the risk of transmission via blood transfusion, we designed this study to survey the seroprevalence of anti-Brucella antibody in blood donors in different endemic provinces of Iran by standard technique protocols.

\section{Materials and Methods}

In this study, exposure to Brucella infection was investigated by serological assay in blood donors from 5 endemic provinces. A total of 14,706 blood donors from 5 different geographical areas were enrolled. The origin and required number of blood donor samples from each province were estimated based on brucellosis seroprevalence data that has been provided by the Iranian Centre for Disease Control (ICDC) (Table 1).

All subjects were asked by a trained physician to complete a questionnaire including demographic data, and their donation and medical history. All donors passed through the interview process without any signs or symptoms of a recent or chronic infec- tion. Serum samples $(2 \mathrm{~mL})$ were taken, and then sent under controlled conditions to the ICDC reference laboratories in each area. All laboratories worked with a unique standard operation program. All samples were tested by standard tube agglutination (STA) and for each positive STA subject, a 2-mercaptoethanol agglutination (2-ME) test was performed.

The STA test was carried out with B. abortus plain antigen provided by the Pasteur Institute of Iran. The sensitivity and specificity of the STA test were 97 and 95\%, respectively (data obtained from the Division of Antibody Production of the kit manufacturer, Pasteur Institute; Wright tube test). B. abortus and B. melitensis have a common epitope in their smooth lipopolysaccharide (SLPS). B. melitensis can therefore be detected by using a complete antigen or by preparing S-LPS from the chemical extraction of $B$. abortus. Serial dilution of the sera in PBS was performed (1:10 to $1: 1,280)$. We added $0.5 \mathrm{~mL}$ of $10 \% \mathrm{~B}$. abortus antigen to each tube and incubation took place at $37^{\circ} \mathrm{C}$ for $24 \mathrm{~h}$. All tubes were compared with antigen control tubes for the degree of opacity of the supernatant fluid. Any serum with a titre $\geq 80$ was considered a positive result and the associated demographic and other potential risk factors were evaluated.

The sensitivity and specificity of the 2-ME test were 97 and $95 \%$, respectively (data was obtained from the kit manufacturer; $2-\mathrm{ME}$ antigen test). The serum treated with $2-\mathrm{ME}$ was tested at the same dilutions as STA. To each tube, $0.5 \mathrm{~mL}$ of $10 \%$ B. abortus antigen was added. Incubation at $37^{\circ} \mathrm{C}$ for $24 \mathrm{~h}$ took place and $2-\mathrm{ME}$ agglutination $\geq 40$ was considered positive.

The main socio-demographic variables were: age, gender, donation type, marital status, occupation, consumption of unpasteurized dairy products, and contact with domestic animals. Donors were categorized, based on their donation history, into 3 types; first-time donors, regular donors, and lapsed donors. Regular donors were those who donated blood more than once a year. A donor with an interval between 2 donations of $>1$ year was categorized as a lapsed donor.

Statistical software was used for data analysis (SPSS v16, SPSS Inc., Chicago, IL, USA). The $\chi^{2}$ and $t$ test were used to detect the significance of differences between data obtained from blood donors and the results of the serological tests. A $p$ value $<0.05$ was considered significant.

\section{Results}

The study population consisted of blood donors from 5 Brucella-endemic provinces in the west and northwest of Iran. A total of 219,929 donors donated blood during the designated period, and 14,706 healthy blood donors 
Table 2. Summary of the socio-demographic characteristics of 14,706 study participants

\begin{tabular}{lc}
\hline & $n(\%)$ \\
\hline Male & $13,571(923)$ \\
Female & $1,135(7.7)$ \\
\hline Donor type & \\
$\quad$ First-time & $6,417(43.6)$ \\
$\quad$ Regular & $4,252(28.9)$ \\
$\quad$ Lapsed & $4,037(27.5)$ \\
\hline Marital status & \\
$\quad$ Single & $4,876(33.2)$ \\
$\quad$ Married & $9,830(66.8)$ \\
\hline Educational status & \\
$\quad$ Post graduate & $2,975(20.2)$ \\
$\quad$ Bachelor & $6,396(43.5)$ \\
College & $4,747(32.3)$ \\
$\quad$ lliterate & $588(4)$ \\
\hline Using unpasteurized dairy products & \\
$\quad$ Yes & $6,957(47.3)$ \\
$\quad$ No & $7,749(52.7)$ \\
\hline Living area & \\
$\quad$ Urban & $13,560(92.2)$ \\
$\quad$ Rural & $1,146(7.8)$ \\
\hline
\end{tabular}

were randomly selected and enrolled in the study. All participants were eligible for blood donation according to the IBTO questionnaire and blood donor selection criteria, and negative for mandatory serologic tests for transfusion-transmitted diseases (HBV, HCV, HIV, syphilis, and HTLV). The age range of participants was $17-70$ years. The mean age of seropositive donors and all participants was $33.5 \pm 10.5$ and $35.8 \pm 10.4$ years, respectively. More than $90 \%$ were male $(n=13,571 ; 92.3 \%)$. Based on the type of blood donation, all were voluntary donors. Descriptive statistics of the 3 specific donor types show that $43.6 \%(6,417 / 14,706)$ were first-time blood donors (Table 2). The majority of them $(9,830[66.8 \%])$ were married. Regarding the level of education, $43.5 \%$ had obtained a bachelor qualification. Most of the donors lived in urban area (92.2\%). Main demographic factors, donation history, and information relating to Brucella exposure appear in Table 2.

STA was reactive in 1,093 sera with different titres, overall seroreactivity by STA (STA $\geq 20$ ) was $7.43 \%$ $(1,093 / 14,706$; $95 \%$ confidence interval [CI] 0.0702$0.0787)$. STA-positivity titres were $20(n=829 ; 5.6 \%), 40$ $(n=201 ; 1.3 \%), 80(n=53 ; 0.3 \%), 160(9 ; 0.06 \%), 320(1$; $0.006 \%$ ), and the remaining $92.7 \%$ were seronegative. By considering STA $\geq 80$ a positive result, a total of $63(0.43 \%)$ serum samples were STA-positive; of these, the 2-ME test was reactive in 42 samples. The 2-ME test was reactive in
Table 3. Summary of serological assay results

\begin{tabular}{ll}
\hline STA $^{\mathrm{a}}$ & $2-\mathrm{ME}^{\mathrm{b}}$ \\
\hline STA-positive $(>80)$ & 2 -ME-positive $(>40)$ \\
$n=63(0.43 \%)$ & $n=11(0.07 \%)$ \\
& 2 -ME-negative $(<40)$ \\
& $n=31(0.21 \%)$ \\
\hline${ }^{\mathrm{a}} 1,093 / 14,706$ screened samples were reactive $(7.4 \%)$. \\
${ }^{\mathrm{b}} 42 / 63$ screened samples were reactive. \\
\hline
\end{tabular}

31 samples with a low titre $(\leq 20)$. However, concomitant STA $\geq 80$ and $\geq 40$ for 2 -ME tests were found in 11 $(0.075 \%)$ cases (Table 3$)$.

Regarding the geographic distribution of the seropositive donors, the highest prevalence was observed in Hamedan (northwestern province), and most positive cases were patients residing in urban areas. Information associated with geographical origin and Brucella seroprevalence among blood donors of different provinces is presented in Table 1. Exposure to manure products was identified as the only significant risk factor for Brucella seropositivity. Seroprevalence and data for each considered risk factors are presented in Table 4.

\section{Discussion}

In an effort to determine brucellosis seroprevalence and its main associated risks for blood safety in the endemic areas, this study conducted a survey of 14,706 blood donors. We found that $63(0.43 \%)$ apparently healthy blood donors were remarkably seropositive. This number is significant, especially considering the fact that $>7 \%$ had evidence of Brucella exposure. This observation was not unexpected, because Iran is located in the eastern Mediterranean region which is endemic for B. melitensis, with wide variations in endemicity in different geographical areas (Fig. 1). In Iran, the annual incidence of brucellosis is estimated at 238.6/1 million of the population [2]. Prevalence in different provinces varies widely, with a highest reported prevalence of 25\% [14]. In this study, the prevalence among blood donors was $0.43 \%$, i.e., much lower than in the general population.

Serological tests for the diagnosis of brucellosis were the STA and 2-ME. Despite variations in interpretation of the STA which measures the anti-polysaccharide $\mathrm{O}$ antibody, it is in common use [15]. Keramat et al. [16] described patients with an STA titre $\geq 80$ or 2 -ME $\geq 40$ as being infected with Brucella. Nowadays, ELISA methods are used to detect IgG and IgM antibodies against the organism [17-20]. The prevalence in our study varied wide- 
Table 4. Risk factors associated with brucellosis seropositivity (STA), comparison between STA-positive and STA-negative cases

\begin{tabular}{|c|c|c|c|c|}
\hline Risk factor & STA <1/80 neg & $\mathrm{STA} \geq 1 / 80 \sim$ pos & $p$ value & RR (95\% CI) \\
\hline Gender & & & 0.9 & $0.97(0.3899-2.4139)$ \\
\hline Male & $13,513(91.9 \%)$ & $58(0.4 \%)$ & & \\
\hline Female & $1,130(7.67 \%)$ & $5(0.03 \%)$ & & \\
\hline $\begin{array}{l}\text { Consumption of unpasteurized } \\
\text { dairy products }\end{array}$ & & & & $1.22(0.7471-2.0127)$ \\
\hline Yes & $6,924(47 \%)$ & $33(0.3 \%)$ & 0.4 & \\
\hline No & $7,719(52.5 \%)$ & $30(0.2 \%)$ & & \\
\hline Donation history & & & 0.19 & - \\
\hline First time & $6,383(43.4 \%)$ & $34(0.23 \%)$ & & \\
\hline Regular & $4,235(28.8 \%)$ & $17(0.11 \%)$ & & \\
\hline Lapsed & $4,025(27.38 \%)$ & $12(0.08 \%)$ & & \\
\hline Marital status & & & 0.19 & $1.45(0.8271-2.5669)$ \\
\hline Single & $4,860(33 \%)$ & $16(0.1 \%)$ & & \\
\hline Married & $9,783(66.6 \%)$ & $47(0.3 \%)$ & & \\
\hline Education status & & & 0.7 & - \\
\hline Post graduate & $2,963(20.14 \%)$ & $12(0.08 \%)$ & & \\
\hline Bachelor & $6,367(43.3 \%)$ & $29(0.2 \%)$ & & \\
\hline College & $4,729(32.15 \%)$ & $18(0.12 \%)$ & & \\
\hline Illiterate & $584(3.98 \%)$ & $4(0.03 \%)$ & & \\
\hline Job & & & 0.34 & $1.49(0.6451-3.4521)$ \\
\hline High-risk & $963(6.56 \%)$ & $6(0.04 \%)$ & & \\
\hline Low-risk & $13,680(93 \%)$ & $57(0.4 \%)$ & & \\
\hline Manure products exposure & & & 0.0128 & $2.35(1.1993-4.6172)$ \\
\hline Yes & $1,101(7.5 \%)$ & $10(0.07 \%)$ & & \\
\hline No & $13,543(92.08 \%)$ & $52(0.35 \%)$ & & \\
\hline Living area & & & 0.9 & $1.02(0.4100-2.5380)$ \\
\hline Urban & $13,502(91.8 \%)$ & $58(0.4 \%)$ & & \\
\hline Rural & $1,141(7.77 \%)$ & $5(0.03 \%)$ & & \\
\hline Animal contact & & & 0.2 & $1.50(0.8040-2.8181)$ \\
\hline Yes & $1,976(13.47 \%)$ & $12(0.08 \%)$ & & \\
\hline No & $12,667(86.1 \%)$ & $51(0.35 \%)$ & & \\
\hline
\end{tabular}

ly across geographical areas, ranging from almost zero in Kurdistan to 18/1,000 blood donations in Hamedan.

Why the prevalence in Kurdistan was low, despite this region having a high seroprevalence, is a topic for future study. We suggest possible reasons. Almost all blood donations from Kurdistan come from the capital of the Kurdistan province, Sanandaj (the Sanandaj Blood Centre). The motivation of Sanandaj residents to donate blood is very high, and the loyal regular donors living in urban areas are aware of their health status. We only considered the titre of 80 as positive for STA agglutination; however, if a lower titre $(\leq 40)$ had been considered, some more positive cases would have been detected amongst the Kurdistan blood donors. The geographical range of infected individuals suggests that most cases were resident in urban areas. This could be due to the fact that the number of blood donors from rural areas is very low; 92.2\% come from urban areas.

To our knowledge, brucellosis seroprevalence among Iranian blood donors was previously evaluated in smallscale studies. Khorasgani et al. [21] studied the largest sample $(n=10,500)$ but all other studies had a much smaller sample than ours. A larger sample size and the focus on provinces with a higher incidence of infection in the general population are the strengths of this study. In 4 previous studies carried out in Iran with research methods similar to ours, the seroprevalence rate was $0.34 \%$ (STA) and $0.20 \%$ (2-ME) in Ahvaz, $0.057 \%$ (STA) in Boushehr, 0.11\% (STA) in Arak, and 6.3\% (STA) and $0.6 \%(2-\mathrm{ME})$ in Yazd [21-24]. The main risk factors for brucellosis in the general population of Iran are: "consumption of unpasteurized dairy products (especially raw 
Fig. 1. Geographical distribution of Brucella seroprevalence in the general population in Iran (used with permission from Mohammad Reza Shirzadi, ICDC, Ministry of Health, Iran).

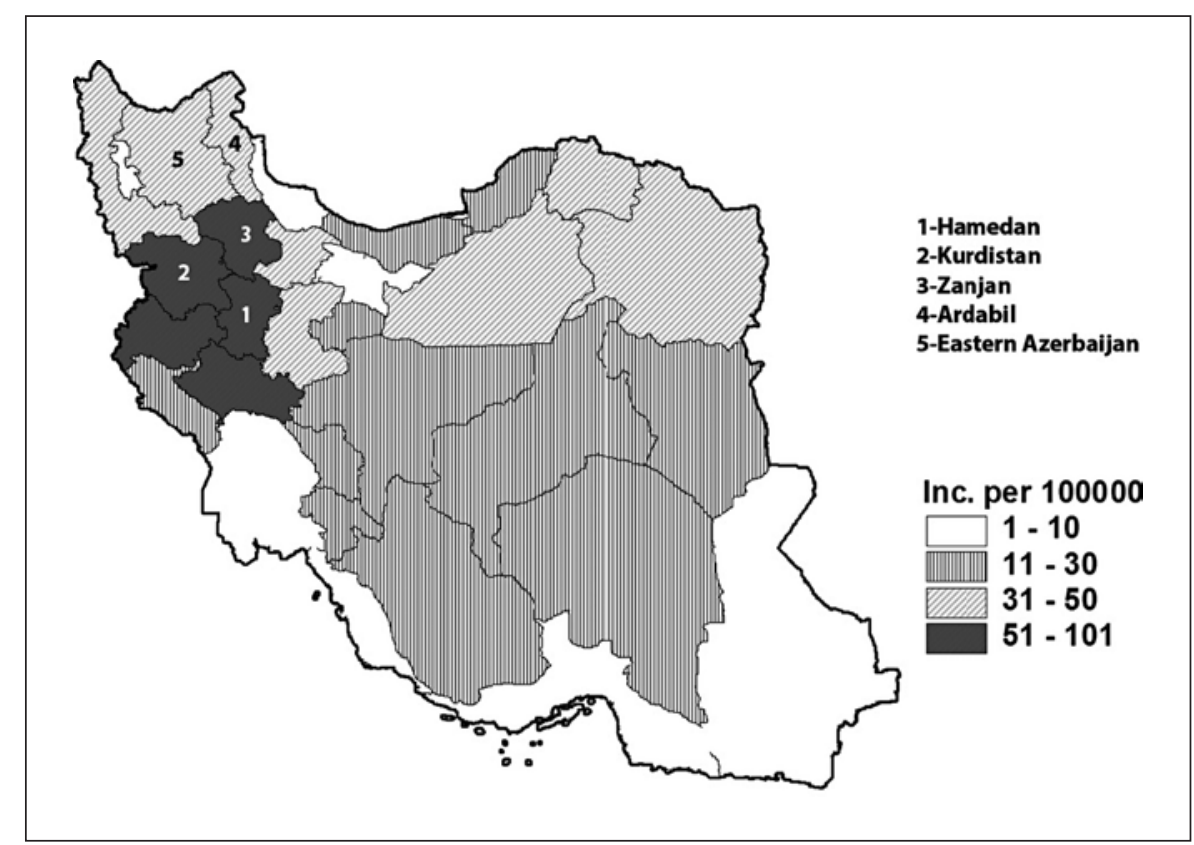

milk and fresh cheese), direct contact with animals and animal husbandry, laboratory and veterinary professions, and the presence of another case of brucellosis at home" [25]. We found a significant association between exposure to manure products and seropositvity. Usually, transmission by ingestion of contaminated dairy products is noticed. It should thus be re-emphasized that other routes of transmission including contact with manure products must be kept in mind. The prevalence of brucellosis among blood donors varies from 0.6 to $3.6 \%$ in different countries such as Turkey [26], Greece [27], India [28], Mongolia [29], and Mexico [30] (0.6, 2.5, 3.11, 3.3, and $3.6 \%$, respectively). The study in Turkey on blood donors of a non-endemic region implemented a molecular detection method (real-time PCR) and found $0.6 \%$ seropositivity by means of STA [26]; a $0.3 \%$ positivity rate was detected by PCR and all blood donors were from rural areas. The authors concluded that this risk was nonsignificant, but that the organism should be evaluated as a threat for blood transfusion.

Another study run in northwestern China revealed that $3.1 \%$ of blood donors had a reactive sample by STA, approximately $0.64 \%$ of which were confirmed serologically by Western blot and $0.39 \%$ by molecular investigation. None were bacteremic by the culture method, and STA-positivity was detected in the circulating DNA of all donors [13]. This data re-emphasizes that most STA positivity in blood donors from endemic regions is not bacteremic and occurs due to past exposure rather than true infection.

A positivity rate of $0.36 \%$ was found in blood donors from an endemic area in India by considering 160 as a significant titre [28]. Another Indian evaluation observed 3.11 and $0.62 \%$ seropositivity among their blood donors by STA and 2-ME, seen particularly in blood donors from rural areas with a history of animal contact or exposure to contaminated dairy products [31].

A study in southern Ethiopia showed a high seroprevalence $(10.6 \%)$ of human brucellosis in blood donors. Contact with domestic animals and husbandry practices at home were the main risk factors [32].

Although a diverse range of brucellosis seroprevalence in Iranian blood donors exist, there have been no reports of blood transfusion-transmitted brucellosis in Iran. We should keep in mind, however, that chronic disease by no clinically apparent infection is possible, and that the intracellular organism can be viable for as long as 6 months at $4{ }^{\circ} \mathrm{C}$, although transmission via the transfusion of stored blood products is also a possibility [7].

Transfusion transmission of Brucella was first described in the 1950s after using whole blood from an asymptomatic but bacteremic donor [33]. In current practice, the transfusion of red blood cells or blood products is more common and whole blood is rarely used. Therefore, the ability of blood components to transmit the disease is the scope of the further investigation.

As there are no tests yet approved for Brucella screening in blood donors and only an imprecise known role of leukoreduction, the following recommendations can be made for further blood transfusion safety in highly endemic areas: pre-donation screening concerning the history of animal contact, the signs and symptoms of acute or chronic febrile disease, and exposure to contaminated dairy products can be considered as the only measure to 
reduce the risk of transfusion-transmitted brucellosis. It is suggested that blood donors should be questioned about the nonspecific symptoms of brucellosis. Moreover, asking about the habits of using the dairy products taken directly from ranchers and modifications applied to possibly contaminated products, along with probing other routes of exposure like direct contact to manure, would be helpful. In addition, we suggest close communication with provincial veterinary organizations to hear about districts with outbreaks of Brucella in livestock, and with local health authorities so as to keep informed of outbreaks in high-risk groups. However, it is difficult to evaluate the usefulness of risk-reduction strategies for brucellosis. It seems that routine deferral of those considered to be at risk has an impact on blood supply in highly endemic areas, but the potential effect would likely not be impressive.

In conclusion, we report a summary of blood donors according to significant serological markers that might pose a risk to blood safety. Further evaluation is recommended to establish the related risk factors, especially in provinces practicing animal husbandry and among donors with a professional contact history. Evaluations for transfusion-transmitted brucellosis, especially in endemic areas, will be helpful for policy-makers. We also recommend further studies using molecular testing on at-risk donors living in endemic areas.

\section{Acknowledgements}

We acknowledge all physicians in the blood donation, sampling, and transportation sections of local provincial centres of the IBTO. We also appreciate the cooperation of the CDC-related laboratory technicians in the given provinces.

\section{Statement of Ethics}

This study was approved by the research ethics committee of Iranian Blood Transfusion Organization (IBTO). All subjects signed an informed consent.

\section{Disclosure Statement}

The authors declare no conflicts of interest.

\section{References}

1 Godfroid J, Cloeckaert A, Liautard JP, Kohler S, Fretin D, Walravens K, et al. From the discovery of the Malta fever's agent to the discovery of a marine mammal reservoir, brucellosis has continuously been a re-emerging zoonosis. Vet Res. 2005 May-Jun;36(3):313-26.

2 Pappas G, Papadimitriou P, Akritidis N, Christou L, Tsianos EV. The new global map of human brucellosis. Lancet Infect Dis. 2006 Feb;6(2):91-9.

3 Mirnejad R, Jazi FM, Mostafaei S, Sedighi M. Epidemiology of brucellosis in Iran: A comprehensive systematic review and meta-analysis study. Microb Pathog. 2017 Aug; 109: 239-47.

4 Meltzer E, Sidi Y, Smolen G, Banai M, Bardenstein S, Schwartz E. Sexually transmitted brucellosis in humans. Clin Infect Dis. 2010; 51(2):e12-15.

5 Mesner O, Riesenberg K, Biliar N, Borstein E, Bouhnik L, Peled N, et al. The many faces of human-to-human transmission of brucellosis: congenital infection and outbreak of nosocomial disease related to an unrecognized clinical case. Clin Infect Dis. 2007;45(12): e135-40.

6 Hasanjani Roushan MR, Ebrahimpour S, Moulana Z. Different clinical presentations of brucellosis. Jundishapur J Microbiol. 2016 Apr;9(4):e33765.
7 Economidou J, Kalafatas P, Vatopoulou T, Petropoulou D, Kattamis C. Brucellosis in two thalassaemic patients infected by blood transfusions from the same donor. Acta Haematol. 1976;55(4):244-9.

8 Polat KY, Tosun MS, Ertekin V, Aydinli B, Emre S. Brucella infection with pancytopenia after pediatric liver transplantation. Transpl Infect Dis. 2012 Jun;14(3):326-9.

9 Akçakuş M, Esel D, Çetin N, Kisaarslan AP, Kurtoğlu S. Brucella melitensis in blood cultures of two newborns due to exchange transfusion. Turk J Pediatr. 2005 Jul-Sep;47(3): 272-4.

10 al-Kharfy TM. Neonatal brucellosis and blood transfusion: case report and review of the literature. Ann Trop Paediatr. 2001 Dec; 21(4):349-52.

11 Pappas G, Panagopoulou P, Christou L, Akritidis N. Brucella as a biological weapon. Cell Mol Life Sci. 2006 Oct;63(19-20):222936.

12 Mense MG, Borschel RH, Wilhelmsen CL, Pitt ML, Hoover DL. Pathologic changes associated with brucellosis experimentally induced by aerosol exposure in Rhesus macaques (Macaca mulatta). Am J Vet Res. 2004 May;65(5):644-52.

13 Wang W, Liao Q, Wu X, Hou S, Wang Y, Wu $\mathrm{J}$, et al. Potential risk of blood transfusiontransmitted brucellosis in an endemic area of China. Transfusion. 2015 Mar;55(3):586-92.
14 Chalabiani S, Khodadad Nazari M, Razavi Davoodi N, Shabani M, Mardani M, Sarafnejad A, et al. The Prevalence of Brucellosis in Different Provinces of Iran during 20132015. Iran J Public Health. 2019 Jan;48(1): 132-8.

15 Al Dahouk S, Tomaso H, Nöckler K, Neubauer H, Frangoulidis D. Laboratory-based diagnosis of brucellosis - a review of the literature. Part II: serological tests for brucellosis. Clin Lab. 2003;49(11-12):577-89.

16 Keramat F, Majzobi MM, Poorolajal J, Ghane ZZ, Adabi M. Seroprevalence of Brucellosis in Human Immunodeficiency Virus Infected Patients in Hamadan, Iran. Osong Public Health Res Perspect. 2017 Aug;8(4):282-8.

17 Ayala SM, Hasan DB, Celestino CA, Escobar GI, Zhao DM, Lucero NE. Validation of a simple universal IELISA for the diagnosis of human brucellosis. Eur J Clin Microbiol Infect Dis. 2014 Jul;33(7):1239-46.

18 Al-Shamahy HA, Wright SG. Enzyme-linked immunosorbent assay for Brucella antigen detection in human sera. J Med Microbiol. 1998 Feb;47(2):169-72.

19 Sanaei Dashti A, Karimi A, Javad V, Shiva F, Fallah F, Alaei MR, et al. ELISA cut-off point for the diagnosis of human brucellosis; a comparison with serum agglutination test. Iran J Med Sci. 2012 Mar;37(1):9-14. 
20 Mantur B, Parande A, Amarnath S, Patil G, Walvekar R, Desai A, et al. ELISA versus conventional methods of diagnosing endemic brucellosis. Am J Trop Med Hyg. 2010 Aug; 83(2):314-8.

21 Khorasgani MR, Esmaeili H, Pourkarim M, Mankhian AR, Salehi TZ. Anti-Brucella antibodies in blood donors in Boushehr, Iran. Comp Clin Pathol. 2008;17(4):267-9.

22 Shakurnia A, Qasemzadeh A, Afra M, Kohshour MO. Sero-prevalence of brucellosis among blood donors in Ahvaz, Southwest Iran. Asian Pac J Trop Dis. 2014;4:S307-10.

23 Sofian M, Sheikholeslami M, Mahdaviani FA, Aghakhani A, Banifazl M, Eslamifar A, et al. Low prevalence of Brucella agglutinins in blood donors in central province of Iran. Iran J Microbiol. 2013 Mar;5(1):24-7.

24 Ghilian R, Hekmati Moghaddam SH, Fatemi A, Eslamieh H, Dargahi M. Seroepidemiologic status of brucellosis in blood donors in Yazd, 2009. Sci J Iran Blood Transfus Organ. 2011;7:196-205.

25 Golshani M, Buozari S. A review of brucellosis in Iran: Epidemiology, Risk Factors, Diagnosis, Control, and Prevention. Iran Biomed J. 2017 Nov;21(6):349-59.

26 Yavuz MT, Kaya D, Behcet M, Aksit H. Detection of Brucella among voluntary blood donors in Turkey by using a new real time PCR method. J Anim Vet Adv. 2012;11(17):324750 .
27 Papadogiannakis E, Kontos V, Kontou I, Kostomitsopoulos N, Siochou E, Tsachev I, et al. A serological survey of brucellosis, echinococcosis, Q-fever, toxoplasmosis, leishmaniasis and Mediterranean spotted fever in animal production employees in Greece. Trakia J Sci. 2007;5:70-8.

28 Vaishnavi C, Kumar S. Investigation for background prevalence of Brucella agglutinins among blood donors. Indian J Med Microbiol. 2007 Jul;25(3):302-4

29 Tserenpuntsag B, Ouynbileg L, Nelson K, McNutt LA. Prevalence of infectious diseases among Mongolian blood donors. J Infect Dev Ctries. 2008 Feb;2(1):73-5.

30 Torres-Padilla JC, López-Merino A, GarcíaEscamilla RM, Gutiérrez-García JN. AntiBrucella antibody seroprevalence in blood donors for therapeutic ends at three blood banks of the Mexican Institute of Social Security. Gac Med Mex. 2004 Jul-Aug;140(4):3918. Spanish

31 Mangalgi S. Sajjan Am, Mohite ST: seroprevalence of Brucellosis among blood donors of STAara district, Maharashtra. J Krishna Inst Med Sci Uni. 2012;1:55-60.

32 Workalemahu B, Sewunet T, Astatkie A. Seroepidemiology of Human Brucellosis among Blood Donors in Southern Ethiopia: Calling Attention to a Neglected Zoonotic Disease. Am J Trop Med Hyg. 2017 Jan;96(1):88-92.

33 Borden CW, Hall WH. Fatal transfusion reactions from massive bacterial contamination of blood. N Engl J Med. 1951 Nov;245(20): 760-5 